\title{
DISTRIBUCIÓN DE INSTALACIONES: MÉTODOS DE SOLUCIÓN Y APLICACIONES RECIENTES
}

FACILITY LAYOUT: SOLUTION METHODS AND RECENT APPLICATIONS

\author{
Aldair A. Álvarez ${ }^{1 *}$, Alfredo D. Moreno ${ }^{1}$, Víctor M. Noble ${ }^{1}$, Jorge M. López ${ }^{2}$ \\ Recibido para publicación: 20 marzol de 2013- Aceptado para publicación: 4 de mayo de 2013
}

\section{RESUMEN}

Este trabajo presenta una revisión del estado del arte del Problema de Distribución de Instalaciones (FLP) y sus distintos métodos de solución, tales como heurísticos, metaheurísticos y exactos. Se hace principal énfasis en técnicas metaheurísticas como Algoritmos Genéticos, Recocido Simulado, Optimización por Enjambre de Partículas, Optimización por Colonia de Hormigas, Búsqueda Tabú y algoritmos híbridos aplicados en los últimos años. Adicionalmente se muestran algunas aplicaciones de métodos de solución de problemas de distribución de instalaciones en diferentes escenarios reales y simulados.

Palabras clave: distribución de instalaciones, heurística, metaheurística.

\section{ABSTRACT}

In this work is presented a state-of-art review of the Facility Layout Problem (FLP) and the its different solution methods, such as heuristic, metaheuristic and exact methods. Principally emphasizing in metaheuristic techniques such as Genetic Algorithm, Simulated Annealing, Particle Swarm Optimization, Ant Colony Optimization, Tabu Search and hybrid algorithm applied in the last years. Additionally some applications are shown of these methods in real and simulated scenarios.

Keywords: facility layout, heuristic, metaheuristic.

\footnotetext{
${ }^{1}$ Ingeniero industrial, Universidad de Córdoba, Montería, Colombia.

2 Master en Ingeniería Industrial, Profesor asociado, Universidad de Córdoba, Montería, Colombia.

*Tel: +57300238 5279, aaad_147@hotmail.com
} 
Aldair Alvarez, Distribucion de Instalaciones

\section{INTRODUCCIÓN}

A lo largo del tiempo se ha estudiado ampliamente como mejorar la productividad y competitividad de las organizaciones. Sule (1994) y Tompkins et al. (1996) argumentaron que la disposición de las instalaciones en una planta es un factor importante para su utilización efectiva y eficiente (Tomado de Matai et al. 2010). Además, hoy en día las organizaciones deben tener la habilidad de adaptarse rápidamente a los cambios (L. Xu et al. 2011). Un factor crítico para conseguir lo anterior es una adecuada distribución en planta teniendo en cuenta los aspectos del costo de manejo de materiales y la adecuada utilización de los espacios. La disposición de instalaciones 0 los elementos productivos (estaciones de trabajo, maquinas, células de trabajo, etc.) dentro del área de trabajo de una planta buscando optimizar alguna función objetivo se conoce como Facility Layout Problem (FLP). La distribución en planta es una problemática que se ha abordado desde diferentes perspectivas que abarcan desde la formulación de modelos matemáticos hasta la aplicación de técnicas metaheurísticas. Desde el punto de vista de la optimización, FLP es un problema del tipo NP-duro, el cual implica, que en general es difícil de resolver (Ohmori et al. 2010b).

En este artículo se realiza una recopilación de los métodos de solución aplicados al problema de distribución de planta en los últimos años y se muestran algunos casos de aplicación encontrados en la literatura.

En la sección 2 se define el problema de distribución en planta y su clasificación. En la sección 3 se realiza una recopilación de las diferentes técnicas heurísticas y metaheurísticas aplicadas al problema en los últimos años. De igual similar, en la sección 4 se muestran algunos casos de aplicación y simulaciones encontradas en la literatura, así como algunas revisiones de literatura hechas en los últimos años sobre el FLP. Por último el la quinta sección se presentan las conclusiones respectivas de la revisión realizada.

\section{MÉTODOS DE SOLUCIÓN}

Hay diferentes métodos para resolver el problema de distribución de planta (FLP) en los sistemas de manufacturas. Estos métodos se pueden clasificar en exactos y aproximados.

Los métodos exactos son aquellos que alcanzan a obtener la mejor solución global del problema. Los algoritmos exactos solo pueden ser aplicados para problemas pequeños encontrando soluciones óptimas en un tiempo razonable, de acuerdo a Bozer y Meller (1997), para problemas con menos de 7 departamentos (Ohmori et al. 2010b). En este grupo de métodos se destacan los algoritmos de ramificaciones y acotaciones (branch and bound), algoritmos de planos de corte (cutting plane algorithms) y combinaciones de estos (branch and cut). Mientras que los métodos aproximados son algoritmos que permiten conseguir soluciones buenas en un tiempo asequible. Entre estos métodos se encuentran técnicas heurísticas y metaheurísticas que están disponibles para resolver problemas de gran tamaño $(n>=20)$ en un tiempo computacional razonable y que se acercan a la solución óptima (Matai et al. 2010).

\subsection{Técnicas heurísticas}

Entre las técnicas de solución del FLP se puede encontrar algunos autores que exponen enfoques heurísticos para la solución de este problema.

Un método heurístico es un procedimiento para resolver un problema de optimización bien definido mediante una aproximación intuitiva, en la que la estructura del problema se utiliza de forma inteligente para obtener una buena solución (Adenso-Díaz et al. 1996).

Cabe aclarar que estos métodos no son tan frecuentes; estos enfoques además, dada su naturaleza heurística no pueden generalizarse para todos los tipos de problemas FLP. A 
continuación se presenta una vista de los más destacados en los últimos años.

Recientemente Navidi et al. (2012) propusieron un nuevo enfoque basado en la teoría de juegos para solucionar el FLP. Trata de una modificación al SA utilizando las premisas del equilibrio puro de Nash y el equilibrio de NashPareto. Este algoritmo fue contrastado con un SA de la literatura obteniendo buenos resultados computacionales. Los autores afirman que es un campo donde todavía falta mucho por investigar.

Por su parte Taghavi y Murat (2011) propusieron un procedimiento heurístico integrado basado en la heurística alterna de Cooper (1963), un algoritmo de perturbación y un heurístico de ubicación secuencial en un modelo de programación mixta entera que ellos proponen. Los resultados experimentales que obtuvieron demuestran que el procedimiento heurístico propuesto es a la vez eficiente y eficaz en la identificación de soluciones de calidad para los problemas de tamaño pequeño, mediano y grande. Además, la estrategia de perturbación es capaz de mejorar aún más la calidad de la solución resultante con sólo $0,8 \%$ y $1,6 \%$ brechas promedio para problemas de tamaño grandes y muy grandes.

En ese mismo año, González-Cruz y GómezSenent Martínez (2011) desarrollaron un método heurístico para resolver el FLP. Este método se basa en el concepto de Entropía (medida del desorden del sistema). La principal diferencia con otras técnicas yace en que la generación y evaluación de alternativas resulta de intercambios en las instalaciones. De modo similar, en 2010 fue desarrollado un método heurístico propuesto por McKendall y Hakobyan (2010) aplicado al DFLP. El procedimiento incluye en uno de sus pasos la modificación de los layout propuestos por medio de búsqueda tabú. Este procedimiento desarrollado demostró que es capaz de encontrar buenas soluciones para el problema en estudio.

Finalmente, Bozer y Wang (2012) diseñan un procedimiento para eliminar las variables binarias de un modelo MIP y así simplificar el mismo. La técnica heurística llamada GRAPH, es un algoritmo basado en recocido simulado que utiliza la técnica de representación por grafos para resolver el modelo MIP. Los resultados muestran la capacidad de la heurística para lidiar de buena manera con problemas encontrados en la literatura.

\subsection{Técnicas metaheurísticas}

En esta parte se muestra un recorrido por algunos trabajos de aplicación y adaptación de metaheurísticas para el FLP y sus diferentes clasificaciones.

La técnicas metaheurísticas Son procedimientos que en un proceso iterativo, guían a una heurística subordinada combinando inteligentemente diferentes conceptos tomados de analogías de la naturaleza y exploran el espacio de soluciones utilizando estrategias de aprendizaje para estructurar la información, con el objeto de encontrar eficientemente soluciones cercanas al óptimo (Osman 2004). Durante los últimos 20 años, las técnicas metaheurísticas han sido aplicadas ampliamente para resolver el problema de distribución de planta (Sadrzadeh 2012).

\subsubsection{Algoritmos Genéticos (GA)}

Existen diversas aplicaciones para el algoritmo genético dentro del campo de la distribución de instalaciones, por ejemplo, Salas-Morera et al. (2011) presentan un algoritmo genético aplicado a la designación de las distribuciones en industrias con instalaciones de áreas desiguales tomando como objetivos la minimización del costo de manejo de materiales, la relación lógica entre espacios y la forma de cada área. Los autores desarrollaron un software que permite a los usuarios introducir los datos de entrada y que representa gráficamente la solución propuesta. Como resultado, se obtuvo que el GA genera buenas soluciones comparadas con otros algoritmos aplicados hasta el momento.

Por otra parte, en su trabajo, Shah et al. (2010), introducen un algoritmo evolucionario para resolver el problema dinámico de 
distribución de instalaciones (DFLP). El problema es tratado con un enfoque multiobjetivo y presentan las distribuciones como un conjunto Óptimo de Pareto para objetivos tanto cuantitativos como cualitativos simultáneamente. Como objetivo cuantitativo buscan minimizar el costo de manejo de materiales (MH cost) entre departamentos. El objetivo cualitativo es maximizar las relaciones de cercanías (CR scores) entre departamentos, basados en satisfacer a las instalaciones que utilizan materiales y personal en común, o en relaciones de adyacencia o separación por razones de seguridad, ruido o limpieza. Los resultados computacionales mostraron que el método propuesto genera mejores soluciones que estudios previos. Además, el método proporciona un amplio conjunto de distribuciones óptimas que proporciona un amplio rango de alternativas, permitiendo que la toma de decisiones acerca de la distribución sea más flexible y basada en las circunstancias.

Asimismo en Jun-lin et al. (2012) proponen unas mejoras al algoritmo genético y utilizan la estructura de árbol de corte basado en el orden de recorrido para formar un sistema de codificación de nuevos cromosomas demostrando orden de distribución, de la relación y la ubicación. Se genera la solución inicial basada en dos principios, a saber, adyacencia de las instalaciones y la generación aleatoria. La estructura del cromosoma se compone de tres secciones en la investigación para que podamos hacer las operaciones genéticas de estas tres secciones respectivamente, y utilizan mecanismos dinámicos y retroalimentación para mejorar la función de penalización. Como resultado de ello, el análisis de los casos típicos demuestra que hay ciertas mejoras en este algoritmo tanto en la eficacia y eficiencia.

Raman (2011) trabajó una metodología integrada que incorpora la variabilidad de los sistemas de manufactura y optimiza la distribución de las instalaciones y el sistema de costo de manejo de materiales (MHS). Utilizan un modelo de colas para modelar la variación del sistema de manufactura y algoritmo genético para resolver el problema de optimización integrado. Como resultado obtienen que el enfoque de optimización integrado puede mejorar significativamente el sistema de producción con respecto al tiempo total de viaje de los materiales, el trabajo en proceso (WIP) acumulado, la utilización y calidad de los equipos de manejo de materiales y el área requerida. Para calcular la distancia entre las instalaciones utilizan el método de medida rectilínea.

Datta et al. (2011) realizaron una aplicación de GA para resolver el SRFLP (Single Row FLP) con el objetivo de minimizar costos. La investigación realizada mostró mejoras con respecto a problemas encontrados en la literatura. Sadrzadeh (2012) presenta un algoritmo genético para resolver el FLP en un sistema de manufactura, donde el patrón de flujo de materiales de multi-líneas es considerado con el de multi-productos. Su objetivo fue minimizar el costo total de manejo de materiales. En este trabajo se utiliza una heurística para generar una población inicial y luego se utiliza algoritmos genéticos para mejorar esta solución. Como resultado se obtuvo que este algoritmo es eficiente resolviendo dos ejemplos con un costo total menor que otros algoritmos genéticos, algoritmos CRAFT y algoritmos basados en la entropía.

L. Xu et al. (2011) proponen la utilización de un algoritmo genético adaptativo para optimizar el costo de manejo de materiales y la utilización de talleres de trabajo dentro del problema de distribución de planta (FLP). El algoritmo propuesto busca optimizar el problema teniendo en cuenta ambas funciones objetivos. Como resultado se muestra que la velocidad y precisión de convergencia del algoritmo genético adaptativo (AGA) es mejor que la del algoritmo genético estándar (GA).

Dentro de los GA se tiene que un parámetro del algoritmo puede afectar de manera significativa los resultados obtenidos, por tanto Hernández Gress et al. 82011) abordaron el FLP con GA realizando diferentes experimentos para determinar los mejores parámetros para solucionar el problema. Se encontró que no existen diferencias entre los resultados. Para la investigación se desarrolló 
un modelo de programación entera mixta (MIP). Otro enfoque estudiado para el FLP dentro del mismo modelo MIP, se encuentra en Ozcelik Y Islier (2011) donde se desarrolló un modelo para generalizar el ULLP (Unidirectional Loop Layout Problem). EI modelo fue testeado con 15 problemas que fueron generados con 10, 20, 30, 40 y 50 estaciones interconectadas en un sistema cíclico de manejo de materiales en el software GAMS. El algoritmo genético propuesto fue codificado en Borland Delphi 6.0. El costo hallado fue menor con el GA y tuvo un tiempo computacional considerablemente menor que los algoritmos de GAMS.

\subsubsection{Recocido simulado (SA)}

Ohmori et al. (2010a) aplican la metaheurística para minimizar el costo de manejo de materiales entre departamentos. Los resultados obtenidos muestran que el algoritmo propuesto puede mejorar los resultados de la función objetivo en comparación con los métodos utilizados previamente para estos problemas. También se puede usar con múltiples objetivos, como en Şahin (2011) donde SE realiza una aplicación para la resolución del FLP con dos objetivos.

Por otra parte, Madhusudanan Pillai et al. (2011) presentan un modelo robusto para el problema de Layout para plantas dinámicas, estas plantas son aquellas en las que una reacomodación de las estaciones de trabajo/máquinas se considera de fácil aplicación y que los costos de reacomodación son bajos, el modelo robusto busca un Layout estable en todos los periodos de producción que optimice los costos de flujo en todos los periodos de modo que no se haga necesaria una reacomodación de la planta, sin dejar a un lado la flexibilidad. También propone un algoritmo SA para el modelo propuesto. Sus resultados los compara con una instancia de la literatura llamada robust celular layout model, los cuales resultan ser muy aproximados. También, Şahin et al. (2010) proponen la aplicación de la metaheurística SA para resolver el problema dinámico de distribución de instalaciones (DFLP) con restricciones de presupuesto. Como resultado de este estudio los autores obtuvieron mejores soluciones en casi todos los problemas con un rango de $1.27 \%$ hasta $6.19 \%$. Adicionalmente Shahbazi (2010)propone un nuevo modelo matemático para el DFLP añadiendo al problema el valor del dinero en el tiempo. Para resolver el problema se utilizan dos algoritmos metaheurísticos que son recocido simulado (SA) y búsqueda tabú (TS). Los resultados muestran que el algoritmo búsqueda tabú es mejor para este tipo de problema de estudio.

En Kia et al.(2011) también se estudia el problema dinámico de distribución en planta; se modela el problema de manufactura celular teniendo en cuenta las condiciones cambiantes del mercado. Se utiliza un modelo MINLP para el problema. Finalmente se comparó una versión extendida de SA con el software Lingo para el modelo dado, pero debido a la complejidad del mismo, el software no fue capaz de encontrar la mejor solución en todos los casos estudiados por lo cual se limitó el tiempo de las corridas a 100hrs. El SA resultó ser mejor que Lingo.

\subsubsection{Optimización por enjambre de partículas (PSO)}

Ficko et al. (2010) propusieron un sistema para el desarrollo del layout en sistemas de manufactura flexibles. El sistema propuesto se compone de un subsistema creativo que puede utilizar diferentes métodos de optimización evolutiva, y un subsistema para evaluar diseños. En el trabajo presentado el subsistema de creación utiliza un método PSO para la creación y modificación de los conjuntos de soluciones. La evaluación de la calidad de la solución se realiza utilizando la búsqueda inteligente de las rutas más cortas de viaje dentro de la disposición.

Otra proposición del PSO al FLP con departamentos de distintas áreas fue la de (Kulturel-konak y Konak (2012) el cual es difícil de resolver de forma óptima. En su investigación se propone un enfoque híbrido de la optimización por enjambre de partículas (PSO) y la búsqueda local para resolver el FLP. Los resultados, los cuales fueron contrastados con casos de la literatura, 
Aldair Alvarez, Distribucion de Instalaciones

muestran que el enfoque propuesto es muy prometedor y capaz de encontrar soluciones optimas anteriormente conocidas en tiempos de CPU muy cortos. Además, encontraron nuevas y mejores soluciones para algunos problemas de prueba.

En Samarghandi et al. (2010) propusieron una versión mejorada de PSO para resolver el SRFLP. Por otra parte, en Jolai et al. (2011) abarcan el problema de localización intra e inter celular en un sistema de manufactura, solucionando el problema utilizando PSO. Mientras que Ohmori et al. (2010b) también abordan el FLP a través de PSO. Los resultados obtenidos para este último, muestran que el método propuesto puede mejorar los resultados de la función objetivo en comparación con los métodos utilizados previamente para problemas de tamaño pequeño.

\subsubsection{Ant Colony Optimization (ACO)}

Wong y Komarudin (2010) solucionan el FLP con áreas desiguales con la metaheurística Ant System (AS) que es una variación del ACO. El algoritmo usa corte de árbol para la representación del Layout. Los resultados muestran mejoras con respecto a los resultados de la literatura. Se muestra la función objetivo a minimizar y algunos parámetros usados en el algoritmo. También, en Li et al. (2011) realizan una aplicación del ACO y una versión mejorada del mismo, para resolver el problema de asignación de $n$ puestos de trabajo a $n$ puestos con exclusiones (EFLP). Se realizó un modelo QAP para el problema, luego se aplicó ACO llegando a la solución óptima del problema estudiado. (Problema pequeño). Los resultados son comparados con el software Lingo.

\subsubsection{Búsqueda Tabú (TS)}

Kothari \& Ghosh (2013) realizan la aplicación de TS al SRFLP. Mientras que Mckendall y Liu (2011) lo utilizan para el problema dinámico de distribución en planta (DFLP). Ellos proponen tres nuevos enfoques metaheurísticos basados en búsqueda tabú (TS) para solucionar el problema. El primero es un algoritmo TS simple, el segundo añade al primero estrategias de diversificación e intensificación y el tercero es un procedimiento TS estadístico. Los resultados mostraron que la segunda metaheurística supera a las otras metaheurísticas propuestas y a las disponibles en la literatura.

\subsubsection{Algoritmos híbridos}

Ozcelik (2012) propone un algoritmo genético hibrido HGA para solucionar el FLP, el cual se compone de una búsqueda local y un GA normal. El algoritmo propuesto se compara con el heurístico MR (Move and Reverse) y GA. Los resultados se compararon con metaheurísticas como ACO, un híbrido de ACO y PSO, el algoritmo de búsqueda dispersa, GA y un algoritmo de TS, así como otras heurísticas y métodos exactos de la literatura. Los resultados experimentales muestran que el algoritmo propuesto es eficaz y eficiente. Se muestra que el HGA es capaz de encontrar las soluciones óptimas para todos los 15 casos conocidos con soluciones óptimas. En los otros 36 casos con desconocidas soluciones óptimas, mejoró las mejores soluciones actuales en la literatura el $56 \%$ de los casos y logro las mejores soluciones actuales para los problemas pendientes.

Adicionalmente Tuzkaya et al. (2011) realizaron un caso de estudio en una fábrica de elevadores, donde aplicaron SA y GA; además aplicaron también un enfoque hibrido de estas técnicas denominado HGASA (por sus siglas en Inglés) en el diseño de las instalaciones de esa empresa, dando como resultados que SA obtuvo mejores soluciones haciendo la comparación calidad de la solución-recursos computacionales usados, en conjunto con HGASA, el cual consumió un poco más de recursos, pero generó soluciones de calidad parecida a SA. Otra aplicación de algoritmos híbridos es la encontrada en Ku et al. (2011), donde hacen un hibrido entre GA y SA para la solución del FLP con formas no fijas. Además, se representa el problema como modelo QAP.

Por otra parte, Shah et al. (2010) presentan un algoritmo genético hibrido de búsqueda local 
(LHGA) para el problema dinámico de diseño de instalaciones (DFLP) incorporando la operación de salto de genes. Como resultado obtuvieron que el algoritmo propuesto LHGA es capaz de presentar un mejor rendimiento que los demás algoritmos evolucionarios y heurísticos con respecto a la calidad de la solución para los problemas de prueba.

Finalmente, Chiang et al. (2011) realizaron un estudio usando TS, PSO y sus combinaciones (TS+PSO, PSO+TS) para abordar el FLP. Los resultados computacionales muestran que los algoritmos de dos etapas (TS+PSO, PSO+TS) son capaces de conseguir mejores resultados en más casos que el TS y el PSO aplicados individualmente. Lo cual demuestra los beneficios de los algoritmos híbridos a la hora de encontrar buenas soluciones.

\section{APLICACIONES Y SIMULACIONES}

\subsection{Aplicaciones}

Desarrollar técnicas para solucionar el FLP es improductivo si no se pudiesen aplicar en la realidad. A continuación se exponen casos de aplicación en la realidad, en los cuales estas técnicas han sido de gran utilidad y se ha evidenciado en sus resultados.

Díaz-ovalle et al. (2010) presentan un nuevo enfoque para determinar la distribución óptima de las instalaciones de procesos. La formulación considera un conjunto de instalaciones ya localizadas y un nuevo conjunto de instalaciones para ser acomodadas dentro del área designada. En este estudio los autores consideran la posibilidad de liberación de tóxicos y asumen valores determinísticos para el viento y las condiciones atmosféricas. El modelo propuesto es formulado como un modelo disyuntivo y convertido en un modelo de programación no lineal mixta entera (MINLP). El modelo es resuelto en el software GAMS. Usando este enfoque se obtiene una distribución más segura que usando un enfoque basado en la mínima distancia. Este enfoque proporciona mayor separación entre instalaciones habitadas e instalaciones con posibilidades de liberación toxica, lo que ocasiona una distribución menos compacta pero segura. Proponen, además, la incorporación de medidas de prevención y mitigación para producir distribuciones más prácticas, compactas y seguras.

En el mismo año Vázquez-román et al. (2010) presentaron un nuevo enfoque la distribución óptima de instalaciones considerando la incertidumbre de la liberación. Esta vez, la formulación propuesta incluye el efecto de la velocidad del viento, la dirección del viento y la estabilidad de la atmosfera para calcular el riesgo de muerte a través de una simulación Monte Carlo. Como resultado en el caso de estudio se obtuvieron múltiples distribuciones óptimas y en general los autores concluyeron que los resultados numéricos demuestran el potencial de este enfoque para mejorar los procesos de distribución de instalaciones.

Wiyaratn y Watanapa (2010) hicieron la aplicación de SLP (Systematic Layout Planning) para mejorar la productividad de una planta procesadora de hierro, reorganizando la planta actual y reduciendo la distancia total que implican los flujos obtenidos al interior de la planta. Del mismo modo Lan y Zhao (2010) propusieron un método de optimización de la distribución de instalaciones teniendo en cuenta tanto factores humanos como logísticos. En este trabajo los autores construyeron actividades de relación entre las zonas de operación y formularon una distribución de instalaciones reduciendo la fatiga de los operadores. El método fue aplicado para mejorar la distribución de las instalaciones de un taller de trabajo de una fábrica optoelectrónica. Esta aplicación muestra que este método es conveniente, efectivo y altamente operativo. Sin embargo, este método solo optimiza la distribución teniendo en cuenta la fatiga de los operarios y no toma en cuenta otros factores humanos.

En 2011 Tuzkaya et al. (2011) realizaron un caso de estudio en una fábrica de elevadores, donde aplicaron las técnicas metaheurísticas de recocido simulado y algoritmo genético; además aplicaron también un enfoque hibrido de estas técnicas denominado HGASA (por sus siglas en Inglés) en el diseño de las instalaciones de esa empresa, obteniendo 
como resultados que la metaheurística recocido simulado obtuvo mejores fitness haciendo la comparación -calidad de la solución - recursos computacionales usados-, en conjunto con HGASA, el cual consumió un poco más pero generó soluciones de calidad parecida a SA. Dentro de los casos de aplicación también encontramos la aplicación que proponen Azadeh et al. (2011) con un algoritmo especial llamado FSFDEA, el cual es un tipo especial de simulación para solucionar un caso especial del Single-Row Facility Layout Problem en el proceso de inyección bajo circunstancias de incertidumbre. Para esta tarea hicieron uso de la simulación de eventos discretos como una poderosa herramienta de análisis de sistemas estocásticos y complejos.

Los casos más actuales en la literatura datan en 2012 cuando Li-fang et al. (2013) utilizaron SLP (Systematic layout planning) para realizar un análisis sistemático del actual problema de distribución de una empresa manufacturera y luego utilizan un nuevo esquema basado en los costos logísticos para optimizar la distribución. Como resultado obtienen que el nuevo esquema basado en costos logísticos es factible y que SLP es indudablemente un método efectivo para resolver el problema de optimización de distribución de instalaciones. Del mismo modo y en el mismo año una aplicación local (Colombia) fue la aportación hecha por Cardona et al. (2012) quienes estudiaron el layout de almacenes pesqueros. Presentaron un estudio analítico del diseño desde el punto de vista de la optimización. También, los autores comparan el desempeño del modelo propuesto y diseños tradicionales, presentando una concepción formal de los ahorros esperados. Los autores se centran en encontrar las condiciones óptimas para la característica más importante del diseño del almacén, que es la pendiente de la diagonal cruzada del pasillo. Las decisiones de diseño se modelan como un problema de optimización no lineal.

Brunese y Tanchoco (2013) estudiaron y destacaron la importancia de las restricciones intra-construcción utilizando un modelo en GAMS y obteniendo como resultado que la inclusión de este tipo de restricciones en el SRMLP conduce a mejorar la calidad de las soluciones obtenidas, además de mejorar el diseño al incluir las consideraciones interiores de los espacios.

\subsection{Simulaciones}

Otra técnica usada para resolver el FLP es el enfoque de la simulación. Este tipo de técnica se destaca por representar analógicamente las situaciones reales.

Amit et al. (2012) propusieron dos alternativas de distribución basadas en SLP para una fábrica de alimentos. Asimismo realizaron una evaluación de las distribuciones propuestas en el software de simulación ARENA. Como resultado obtuvieron que la distribución propuesta, era capaz de mejorar la distancia espacial entre maquinas, entre estaciones de trabajo y entre sitios directamente relacionados a la serie del proceso de producción. Con estas reducciones de espacio el manejo de materiales entre instalaciones tomaba menos tiempo, los trabajadores se movían más rápido y la productividad aumentaba, lo que reduce los costos variables de la operación diaria.

Una de las aplicaciones más importantes de un buen layout, el cual acrecienta su importancia es la seguridad pasiva. Carvalho et al. (2011) estudiaron la disposición de los asientos dentro de los vagones de carga de un ferrocarril y su impacto en la seguridad de los pasajeros; esto lo realizaron mediante el uso de simulación, el modelo de la simulación fue codificado y testeado en el software MADYMO. Los resultados mostraron que el layout interno de los vagones afecta significativamente la seguridad de los pasajeros e identificaron los puntos más propensos a lesiones en los casos de choques y accidentes. Por su parte Zhenyuan et al. (2011) abarcaron teoría de la relación entre la forma cómo están distribuidos los espacios y la productividad ya que, según ellos, muchas industrias en China presentan este problema. Modelaron el problema con dimensiones fijas y distancias Manhattan entre centroides. Finalmente se hace una simulación del modelo propuesto mostrando mejoras en la productividad. Shahin y Poormostafa (2011) 
Aldair Alvarez, Distribucion de Instalaciones

también utilizan técnicas de simulación y de jerarquía para abordar el FLP.

\subsection{Otros métodos, aplicaciones y problemas de distribución de instalaciones}

Además de las técnicas metaheurísticas, heurísticas y exactas vistas en capítulos anteriores y de los métodos y problemas que se han considerado existen otros los cuales se han trabajado en los últimos años.

Chung y Tanchoco (2010) estudian como disponer estaciones o maquinas en ambos lados de un corredor en un problema de Double Row Layout Problem. Estos autores hacen la comparación entre 5 heurísticas y CPLEX, mostrando un modelo MIP propuesto para el problema. Como resultado obtienen que las cinco heurísticas desarrolladas bajo este modelo son capaces de encontrar buenas soluciones rápidamente, las cuales pueden servir como soluciones iniciales para algoritmos de corte y ramificación o algoritmos de mejora.

En este mismo año Wong et al. (2010) aplican otro modelo MIP, pero esta vez aplicado al establecimiento de áreas claves dentro de una construcción en proceso. Estos autores comparan el modelo MIP y un algoritmo genético y muestran que el modelo MIP es ligeramente mejor que la metaheurística.

Más adelante Maniya y Bhatt (2011) presentan un nuevo método sistemático y alternativo para la selección de problemas de distribución de instalaciones basado en el método del índice de selección de preferencia (PSI). Este estudio tiene como conclusión que la metodología de selección de distribución de instalaciones basada en el método PSI es simple, lógica y apropiada para resolver el problema y permite evaluar y seleccionar la distribución optima dentro de varias alternativas dadas.

Hongxia y Pengcheng (2011) también presenta un nuevo método para abordar el problema de distribución de instalaciones. Este método considera el espacio de diseño como un polígono y emplea un algoritmo heurístico dividiendo el problema en dos pasos: primero decidir cuál rectángulo va a ser ubicado y segundo en qué posición va a ser ubicado.
Como resultado obtienen que el método que proponen es eficiente para resolver el problema de diseño de instalaciones rectangulares.

Ulutas y Sarac (2011) desarrollan una investigación respecto al algoritmo MSG encontrando los parámetros aplicados al problema de DFLP en el modelo propuesto por Balakrishnan y Cheng (1998). Como resultado obtuvieron que el MSG es un algoritmo eficiente y competitivo aplicado al problema DFLP.

Akbari Jokar y Shoja Sangchooli (2011) proponen un método basado en teoría de grafos para resolver el FLP. Los resultados están a la vanguardia porque son capaces de tener las dimensiones de las instalaciones como entradas, lo cual hace más práctica las soluciones.

En 2012 encontramos otros métodos y aplicación importantes para el problema de distribución de instalaciones. Venkata Rao y Singh (2012) y Hale et al. (2012) proponen métodos que utilizan la distancia entre los centroides de las estaciones. Los primeros proponen un método para evaluar la calidad de la solución con base en la distancia euclidiana entre la solución encontrada y la solución ideal y la anti-ideal. La distancia se valora de acuerdo a los factores a evaluar con su ponderación asignada. Los autores muestran las fórmulas para estandarizar los valores de los criterios y el cálculo de los pesos. Mientras que los últimos proponen un método de construcción de layout mejorado que se basa en la distancia centroide a centroide de las instalaciones. El enfoque utiliza la premisa de que las reducciones en los términos de error centroide a centroide basados en la distancia conducirán a una mejor (y veces más manejable) solución a un problema.

Haktanirlar Ulutas y Kulturel-Konak (2012) estudian el FLP con áreas desiguales y formas no fijas usando la metaheurística AIS (Artificial Inmune Systems) la cual imita el sistema inmunológico. Los resultados encontrados por los autores mejoran en su mayoría los resultados encontrados en la literatura hasta el momento. 
Ripon et al. (2012) estudian el FLP multiobjetivo con áreas desiguales con la técnica VNS. Los objetivos que utilizan son: minimizar el costo de manejo de materiales y maximizar la adyacencia de las locaciones de acuerdo a la importancia de su proximidad o tasa de proximidad (CR). El enfoque VNS es capaz de encontrar un frente óptimo de solución, el cual le da libertad al tomador de decisiones para elegir la alternativa de distribución más adecuada a las necesidades del mercado.

Altuntas y Selim (2012) proponen un nuevo enfoque para el problema de distribución en planta basado en la regla de asociación de pesos. En el enfoque de regla de asociación basado en pesos, a cada ítem es asignado un peso de acuerdo a su significancia con respecto a algún criterio definido por el usuario. En este artículo los autores muestran varios métodos de asociación de pesos, llamados MINWAL(O), MINWAL (W), WARM and BWARM. Los criterios utilizados son la demanda, factor de manejo de partes y eficiencia del equipo de manejo de materiales. Este estudio se diferencia de los anteriores en que, tienen en cuenta estos tres factores juntos. Los resultados indican que el enfoque WARM provee mejores resultados en términos de cantidad total de productos producidos, tiempo de ciclo, tiempo de espera en cola y tiempo de transferencia.

Hadi-Vencheh y Mohamadghasemi (2012) realizan un estudio de FLP modelándolo como NLP, y resolviéndolo mediante un proceso analítico jerárquico teniendo en cuenta múltiples criterios para evaluar la calidad de la solución dada. Muestran el modelo propuesto y destacan que el modelo busca evaluar la calidad de la solución con base en los criterios establecidos, pero no encuentra las soluciones a evaluar.

Chung y Tanchoco (2010) propusieron un modelo de programación entera mixta para el double row layout problem (DRLP), el cual Zhang y Murray (2012) modificaron realizando algunas correcciones al mismo.

\subsection{Revisiones de literatura}

A lo largo de los últimos años muchos autores han trabajado diferentes clases de problemas de distribución en planta, desarrollando nuevos métodos, enfoques y soluciones para el problema. En algunas investigaciones los autores hacen una exhaustiva revisión de la literatura sobre diferentes problemas de FLP.

Drira et al. (2007) presentan una estructura general para analizar la literatura y trabajos existentes de FLP. En este trabajo los autores utilizan como criterio las características de los sistemas de manufactura, las consideraciones dinámicas 0 estáticas del problema, la representación continua o discreta, la formulación del problema y los enfoques de resolución.

Kundu y Dan (2010) al igual que Matai et al. (2010) presentan una investigación sobre los modelos usados para resolver el FLP.

Kundu y Dan presentan un análisis de los modelos que representan el FLP, desde el modelo QAP hasta el modelo multi-plant. Luego muestran los distintos objetivos que se pueden tener a la hora de resolver el problema por medio de GA. Adicionalmente se hace mención sobre otras técnicas distintas a los GA para resolver el problema tales como las técnicas Branch and Bound, SA, TS, PSO y ACO. Por su parte Matai, Singh y Mittal realizan una revisión en general de los distintos modelos matemáticos usados para resolver el problema.

Por último, Hungerländer y Rendl (2012) realizan una comparación de los diferentes enfoques para el problema de single-row facility layout (SRFLP) y aplican un enfoque de programación semi-definida (SDP) para problemas cuadráticos en general. Los autores reportan soluciones óptimas para muchas instancias encontradas en la literatura con más de 42 departamentos, que no habían sido resueltas hasta ahora. Otro resultado importante es que mejoran las soluciones y tiempos de corridas para grandes instancias con más de 110 departamentos. 
Aldair Alvarez, Distribucion de Instalaciones

\section{CONCLUSIONES}

problema de la distribución de instalaciones ha sido la base para el desarrollo de todas las teorías expuestas en la literatura. En torno al FLP hay muchas investigaciones desarrolladas y se han creado técnicas para darle solución a este problema que varían desde los métodos exactos hasta métodos aproximados y simulaciones. No obstante es un campo reciente y falta mucho por explorar en lo que a investigación se refiere.

La investigación reciente evidencia la demanda de nuevos algoritmos que sean capaces de encontrar soluciones eficientes en tiempos aceptables y no sólo esto, sino también que dichas soluciones sean más realistas, lo que le da a la modelación un papel fundamental a la hora de tener todas estas consideraciones y factores en cuenta.

Las tendencias apuntan entonces a que la investigación se realice dividiendo el rumbo del FLP en dos vías paralelas: la primera incluye el desarrollo de nuevos modelos matemáticos que intenten envolver y representar la realidad del FLP. El segundo camino por su parte tendrá que ver con la búsqueda de algoritmos que sean capaces de encontrar soluciones buenas en tiempos aceptables para problemas grandes de FLP. Esto pone a la modelación matemática y a la implementación de técnicas heurísticas y metaheurísticas en el camino a seguir para futuros trabajos en este campo.

\section{REFERENCIAS}

[1]. Adenso-Díaz, B. et al., 1996. Optimización Heurística y Redes Neuronales en Dirección de Operaciones e Ingeniería, Madrid: Paraninfo.

[2]. Akbari Jokar, M.R. \& Shoja Sangchooli, A., 2011. Constructing a block layout by face area. International Journal of Advanced

Manufacturing Technology, 54(5-8), pp.801-809. Available at: http://www.springerlink.com/index/10.1007/ s00170-010-2960-4 [Accessed February 3, 2013].
[3]. Altuntas, S. \& Selim, H., 2012. Expert Systems with Applications Facility layout using weighted association rule-based data mining algorithms : Evaluation with simulation. Expert Systems With Applications, 39(1), pp.3-13. Available at: http://dx.doi.org/10.1016/j.eswa.2011.06.0 45 [Accessed February 3, 2013].

[4]. Amit, N. et al., 2012. Using Simulation to Solve Facility Layout for Food Industry at XYZ Company. Symposium on Humanities, Science and Engineering Research. IEEE, pp.647-652.

[5]. Azadeh, a. et al., 2011. An integrated fuzzy simulation-fuzzy data envelopment analysis algorithm for job-shop layout optimization: The case of injection process with ambiguous data. European Journal of Operational Research, 214(3), pp.768-

779. Available at: http://linkinghub.elsevier.com/retrieve/pii/S 0377221711004401 [Accessed February 4, 2013].

[6]. Balakrishnan, J. \& Cheng, C.H., 2009. The dynamic plant layout problem: Incorporating rolling horizons and forecast uncertainty. The International Journal of Management Science, pp.165-177.

[7]. Bozer, Y. a \& Wang, C., 2012. A graph-pair representation and MIP-model-based heuristic for the unequal-area facility layout problem. European Journal of Operational Research, 218(2), pp.382-391. Available at:

http://dx.doi.org/10.1016/j.ejor.2011.10.052 [Accessed February 3, 2013].

[8]. Brunese, P.A. \& Tanchoco, J M A, 2013. On implied within-building constraints for machine layout. International Journal of Production Research, pp.1937-1952.

[9]. Cardona, L.F., Rivera, L. \& Martínez, H.J., 2012. International Journal of Logistics Research and Applications: A Leading Journal of Supply Chain Management Analytical study of the Fishbone Warehouse layout. International Journal of Logistics Research and Applications: A 
Leading Journal of Supply Chain Management, pp.365-388.

[10]. Carvalho, M., Ambrosio, J. \& Milho, J., 2011. Implications of the inline seating layout on the protection of occupants of railway coach interiors. International Journal of Crashworthiness, pp.557-568.

[11]. Chiang, W.-C. et al., 2011. Two-Stage Tabu - Particle Swarm Algorithms for the Facility Layout Problem with Size Constraints. In IEEE Congress on Evolutionary Computation. pp. 1679-1686.

[12]. Chung, J. \& Tanchoco, J.M.A., 2010. The double row layout problem. International Journal of Production Research, 48(3), pp.709-727. Available at:

http://www.tandfonline.com/doi/abs/10.108 0/00207540802192126

[Accessed February 3, 2013].

[13]. Datta, D., Amaral, A.R.S. \& Figueira, J.R., 2011. Single row facility layout problem using a permutation-based genetic algorithm. European Journal of Operational Research, 213(2), pp.388$394 . \quad$ Available at: http://linkinghub.elsevier.com/retrieve/pii/S 0377221711002712 [Accessed February 3, 2013]. 